\title{
Simulation and Analysis of Dynamic Regimes Involving Ground and Excited State Transitions in Quantum Dot Passively Mode-Locked Lasers
}

\author{
Tianhong Xu, Mattia Rossetti, Paolo Bardella, and Ivo Montrosset, Member, IEEE
}

\begin{abstract}
We present a modified version of the multisection delayed differential equation model, for quantum dot passively mode-locked (ML) lasers when competition between ground state (GS) and excited state (ES) ML takes place. The model takes into account the difference in the group velocity of GS and ES fields. Sole GS, sole ES, and dual-state lasing and ML have been studied. The results are verified with time domain traveling wave simulations and compared, when possible, with experimental results. These tests confirmed the reliability of the model. We found that, in two-section ML lasers, GS lasing and mode locking are always more easily established. For instance, GS lasing can be either self-starting or induced by the initial lasing from the higher energy ES. On the contrary, GS lasing tends to inhibit, to a certain extent, the onset of ES lasing, especially at low injection current and low reverse voltage. Moreover, ES shows less potential to achieve stable ML than GS. Based on these findings, we propose proper theoretical explanation of the achieved lasing and ML regimes in realized devices. Especially, we demonstrate a novel stable dual-state ML regime with remarkable enhanced pulse peak power and pulse width.
\end{abstract}

Index Terms-Delayed differential equations, mode locked lasers, modeling, quantum dot lasers.

\section{INTRODUCTION}

A S A VERY promising candidate for generating stable, picosecond or subpicosecond optical pulses, monolithic passively mode locked (ML) quantum dot (QD) semiconductor lasers have attracted increasing attention in last ten years [1]-[3]. During the development of such devices, various lasing regimes, and in particular ML operating regimes have been observed experimentally [4]-[6], such as the sole ground state (GS) ML, the most recently investigated sole excited state (ES) ML [7] and the dual-state (GS and ES) ML [8]. The last two ML regimes were found always in the devices with active region consisting of few stacked layers of self-assembled QDs, since ES gain is still well below threshold when GS lasing turns on in devices with 10 or 15 QD layers. Therefore in this analysis we consider devices with only $5 \mathrm{InAs} / \mathrm{InGaAs}$ QD layers.

Manuscript received March 23, 2012; revised June 5, 2012; accepted June 25, 2012. Date of publication June 27, 2012; date of current version July 11, 2012. This work was supported in part by the European Community's Seventh Framework Programme (FP7/2007-2013) under Grant 224338 (FAST-DOT Project).

The authors are with the Dipartimento di Elettronica e Telecomunicazioni, Politecnico di Torino, Turin 10129, Italy (e-mail: tianhong.xu@polito.it; mattia.rossetti@polito.it; paolo.bardella@polito.it; ivo.montrosset@polito.it).

Color versions of one or more of the figures in this paper are available online at http://ieeexplore.ieee.org.

Digital Object Identifier 10.1109/JQE.2012.2206372
Unlike the generally observed GS ML which has already been comprehensively studied [1], detailed theoretical investigations of the dual-state ML regime are still not presented so far. A full understanding of the complex dynamics happening in the gain and the saturable absorber (SA) sections has been found to be essential in explaining this novel operating regime. Recently, reverse-emission-state-transition and the two-state ML regime in a strongly chirped QD laser under very low reverse bias voltage of the $\mathrm{SA}(0 \mathrm{~V}$ to $-1 \mathrm{~V})$ were demonstrated experimentally and theoretically in [7]. Instead of the large time consuming time-domain-travelingwave (TDTW) model used in [7], a more efficient numerical model is exploited in this work, enabling a more detailed and wider investigation of the dual-state ML regime in QD two-section ML lasers.

To correctly describe the dynamics of the optical fields generated from two states with distinct wavelengths, it is essential to include also the group velocity difference between these two fields in the numerical model. In Section II, we present a modified version of the multi-section delayed differential equation (MS-DDE) model proposed in [9], which guarantees sufficient accuracy and high computational efficiency at the same time. These qualities are validated through the comparison with the more rigorous TDTW model by performing simulations for the same device in a reduced set of operation conditions.

In Section III, the static and dynamic behaviors of the gain and absorption at GS and ES wavelengths are reported. These results clearly show that some commonly observed advantages of QD active medium, which are also the key factors to obtain stable and high quality ML from the QD GS, are weakened to a certain extent for ES ML. In addition, we found that, for two-section lasers, the GS pulse-induced gain and absorption changes tend to prevent partially the possibility of lasing from higher energy states (ES in this work). The ES pulseinduced gain and absorption variations on the contrary favor the onset of GS lasing. Both effects are more pronounced at low injection current and low SA voltage. These physical considerations allow to give a clear interpretation of the device performances discussed in the following sections.

In Section IV, the simulation results of the lasing and ML operating regimes of two passively ML lasers with different intrinsic cavity losses are shown and discussed. In particular, we show that, at relatively low reverse bias voltages and low gain currents, unstable dual-state ML with slow amplitude modulation of the pulse amplitude is obtained, as found 
experimentally in [7], [8]. On the contrary, when we increase both the voltage and the current, a stable dual-state ML is achieved which, to best of our knowledge, has not been reported in literatures yet. Especially, in the latter case, simultaneous GS and ES MLs are achieved with the same repetition rate for both states, and with significantly enhanced pulse peak power and pulse width.

In Section V, we explained the difficulties to get sole ES ML with respect to the typically observed sole GS ML, based on the information presented in Section III. Finally, a brief conclusion is drawn in Section VI.

\section{NumericAl Model}

A typical two-section passively ML Fabry-Perot (FP) laser, consisting of a forward biased gain section and a reverse biased saturable absorber section, is schematically shown in Fig. 1(a). To model this kind of device using the MS-DDE model proposed in [9], an equivalent unidirectional ring cavity is assumed with the total length $L^{R i n g}=2^{*} L^{F P}$, as shown in Fig. 1(b).

With respect to the TDTW model [10], the DDE model has several distinguished advantages in terms of computational cost and simplicity allowing large parametric analysis of ML lasers. However, the fundamental underlying hypotheses, which enable its high efficiency, also reduce in part its accuracy. In the standard DDE model [11], a reference framework moving at the same group velocity of the pulse is assumed, by considering the following coordinate change $(t, z)$ to $\left(\tau=t-z / v_{g}, z\right)$. With this assumption, the complex field dynamics in the laser cavity can be simply represented just by the field at the reference section $z=0$ and the slowly varying field envelope $E(\tau)$ in the reference section is governed by the following equation:

$$
\frac{d E}{d \tau}(\tau)=-\gamma E(\tau)+\gamma R(\tau-T) E(\tau-T)
$$

where $T$ is the cold cavity round trip time given by $T=$ $L^{\text {Ring }} / v_{g}$ with $v_{g}$ the group velocity in the waveguide, $\gamma$ is the width of a Lorentzian function representing the filtering effect in the laser cavity and $R(\tau)$ represents the round trip gain or losses experienced by the field within the cavity.

Using this approach, all the distributed mechanisms such as the modal gain, the intrinsic losses, and the filtering effect due to finite gain spectrum bandwidth are modeled as lumped elements, limiting the accuracy of this model. The MS-DDE proposed in [9], partially overcomes this problem by considering the laser cavity composed by an arbitrary number $F$ of electrically isolated sections (Fig. 1(b)). In this way, the modal gain and the unsaturable losses are considered distributed in a certain sense. As being compared in detail with the results from TDTW in [9], this MS-DDE model allows reliable and more quantitative description of the QD ML laser with respect to the standard DDE model and its low computational demand is preserved at the same time.

In this work, to take into account the desynchronization of ES and GS pulses due to the different group velocities, we approximated it as a lumped effect at the reference section. Thus, in this improved version of MS-DDE, two delayed

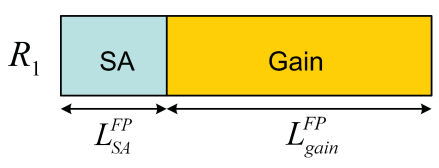

(a)

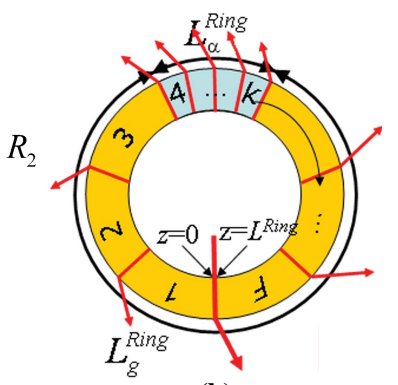

(b)
Fig. 1. (a) Schematic of the considered two-section passively ML FP laser and (b) equivalent unidirectional ring laser described by the proposed MS-DDE model. Forward biased gain section (yellow) and the reverse biased saturable absorber (light blue) are highlighted. The simulated device (b) consists of $\mathrm{F}$ isolated sections; red lines/arrows indicate the nonsaturable losses introduced at each interface between adjacent sections.

differential equations with different delay times are exploited, governing the time-domain evolution of the field envelope of GS and ES respectively:

$$
\begin{aligned}
& \frac{d E_{G S}}{d \tau}(\tau)=-\gamma E_{G S}(\tau)+\gamma R_{G S}\left(\tau-T_{G S}\right) E_{G S}\left(\tau-T_{G S}\right) \\
& \frac{d E_{E S}}{d \tau}(\tau)=-\gamma E_{E S}(\tau)+\gamma R_{E S}\left(\tau-T_{E S}\right) E_{E S}\left(\tau-T_{E S}\right)
\end{aligned}
$$

where parameters have the same meanings as in (1) and the additional subscripts are used to distinguish the corresponding confined QD state.

The round trip gain of GS/ES state $\left(R_{G S} / R_{E S}\right)$ in (2) is calculated using the associated rate-equation system, which describes the carrier distribution dynamics in the QD medium. Due to the inherent hypothesis behind the DDE approach, $R_{G S}$ and $R_{E S}$ are computed assuming that the two fields are propagating with the same group velocity of the ES $v_{g_{-} E S}$, within the cavity. The relative delay between these two fields, being $T_{G S}$ different from $T_{E S}$, is introduced only as a lumped element at $z=0$ to describe the field desynchronization. This approximation is possible because the difference between $T_{G S}$ and $T_{E S}$ is indeed less than $1 \mathrm{ps}$, so comparable with the typical QD carrier relaxation time in the sub-picosecond range [12], [13] and much shorter than the carrier radiative or non-radiative recombination time (hundreds ps to several nanoseconds).

Description of other parts of this DDE model and the rateequation system can be found in [9] and [10].

In order to clarify its correctness, the model has been first used to simulate the two-section ML laser with highly chirped QD active region in [7], very similar dynamic regimes and pulse performances were observed with approximately a 20 -fold decrease in the simulation time. Furthermore, successful simulations were also done with FDTW to verify some cases examined in this paper. Therefore, although this MS-DDE model is not as rigorous as the complex TDTW model [10], we believe the main mechanisms in a QD twosection laser with dual-state lasing are properly considered in it. Consequently, an extensive analysis of the dependence 
of ML regimes on the device cavity and bias parameters can be efficiently preformed with this revised MS-DDE model.

\section{GAin AND AbSorption Dynamics}

The model parameters used in the rate-equation system for computing interband and intraband carrier transitions, are reported in Table I. We consider a device with active region consisting of $5 \mathrm{InAs} / \mathrm{In}_{.15} \mathrm{Ga}_{.85}$ As Dots-in-a-Well (DWELL) layers and emitting around 1.3/1.18 $\mu \mathrm{m}$ from GS/ES. In this section, both the steady-state and the dynamic properties of the gain and the absorption for GS and ES are studied, under different bias and excitation conditions. This preliminary analysis will be essential for discussing and understanding the significant differences between GS and ES lasing and ML regimes achieved in the two-section QD ML lasers studied in Section IV.

\section{A. Steady State Behaviors}

Fig. 2(a) shows the steady state evolution of the material gain from the GS $\left(g_{G S}\right)$ and ES $\left(g_{E S}\right)$ transitions as a function of the injection current density $J$. These results are calculated by searching for the steady state solution of the rate-equation system [10] under different $J$, when assuming no optical field is present (so without optical power-induced gain/absorption depletion). The same parameters are depicted also in Fig. 2(b) but as a function of the corresponding averaged carrier density $n$ in the entire DWELL layers (divide the total carrier number in the quantum well and QD states by the volume of DWELL layers). The behaviors of the corresponding differential gains $d g_{p} / d n(p=\mathrm{GS}$, ES) are also shown in Fig. 2(b). For semiconductor devices, the rate of change of the gain with the total carrier density $d g_{p} / d n$ generally decreases when $n$ increases due to the limited density of available states. Consequently, the material gain will remain almost constant in a wide range of high injection current density. This well-known gain saturation effect is achieved at much lower pumping current density in QD device with respect to its bulk and quantum well counterparts due to the reduced density of states. As expected from above considerations, early GS gain saturation at moderate pumping level is observed in Fig. 2(a). In addition, a much higher GS differential loss $d \alpha_{G S} / d n$ in the absorption regime (when $g_{G S}<0 \mathrm{~cm}^{-1}$ ) with respect to the differential gain $d g_{G S} / d n$ in the gain regime (when $g_{G S}>0 \mathrm{~cm}^{-1}$ ) is highlighted in Fig. 2(b). However, it is also clearly shown that these properties are weakened for the ES, where the material gain, although much higher, has a significantly reduced $d \alpha_{E S} / d n$ and a higher $d g_{E S} / d n$ at the usual operating current density level.

This big difference between GS and ES differential gains/losses has an important influence on the achievement of stable ML pulses from these two transitions in the passively mode locked lasers.

To achieve a stable ML, correct balance should be found between the pulse shortening and attenuation effects in the SA and the pulse broadening and amplification effects in the gain section. Several fundamental conditions therefore need to
TABLE I

Main Model Parameters Used in the Rate Equations

\begin{tabular}{|c|c|c|}
\hline Symbol & Description & Values \\
\hline$H_{w}$ & QW width & $5 \mathrm{~nm}$ \\
\hline$v_{g G S}$ & GS group velocity & $8.967 \cdot 10^{7} \mathrm{~m} / \mathrm{s}$ \\
\hline$v_{g E S}$ & ES group velocity & $8.772 \cdot 10^{7} \mathrm{~m} / \mathrm{s}$ \\
\hline$N_{l}$ & number of QD layers & 5 \\
\hline$N_{D}$ & QD surface density & $2.7 \cdot 10^{10} \mathrm{~cm}^{-2}$ \\
\hline $2 \hbar \gamma$ & gain spectral bandwidth & $34 \mathrm{meV}$ \\
\hline$\Gamma_{x y}$ & field confinement factor & $5.24 \%$ \\
\hline$D_{p}$ & $\begin{array}{l}\text { state degeneracy } \\
p=E S_{2}, E S, G S\end{array}$ & $6,4,2$ \\
\hline$g_{0 p}$ & $\begin{array}{l}\text { material gain coefficient } \\
p=E S, G S\end{array}$ & $379.4,227.6 \mathrm{~cm}^{-1}$ \\
\hline$\tau_{c}^{p}$ & $\begin{array}{l}\text { relaxation times } \\
p=W L, E S_{2}, E S, G S\end{array}$ & $\begin{array}{l}\text { Gain section: } \\
24.2,0.3,0.2,0.2 \mathrm{ps} \\
\text { SA section: } \\
0.3,0.3,0.2,0.2 \mathrm{ps}\end{array}$ \\
\hline$\tau_{r}^{p}$ & $\begin{array}{l}\text { interband recombination times } \\
\text { from } p=S C H, W L\end{array}$ & $400,400 \mathrm{ps}$ \\
\hline$\tau_{s}^{p}$ & $\begin{array}{l}\text { spontaneous emission } \\
\text { recombination } \\
\text { times from } p=E S_{2}, E S, G S\end{array}$ & $2,2.7,2.7 \mathrm{~ns}$ \\
\hline$\tau_{A u}^{p}$ & $\begin{array}{l}\text { Auger recombination times } \\
\text { from } p=E S_{2}, E S, G S\end{array}$ & $110,275,660 \mathrm{ps}$ \\
\hline$\hbar \omega_{p}$ & $\begin{array}{l}\text { interband transition energies for } \\
p=E S_{2}, E S, G S\end{array}$ & $1.114,1.054,0.9879 \mathrm{eV}$ \\
\hline$V_{b i}$ & $\begin{array}{l}\text { built-in potential of the p-i-n } \\
\text { junction }\end{array}$ & $-0.8 \mathrm{~V}$ \\
\hline
\end{tabular}

be satisfied [14]. Among them, the well-known $S$ parameter has been verified to play an important role in determining the performances of the ML systems [15]. It is defined as:

$$
S_{p}=\frac{E_{p, \text { sat }, \text { gain }}}{E_{p, \text { sat }, S A}}=\frac{\left.\left(d \alpha_{p} / d n\right)\right|_{n_{p}=0}}{\left.\left(d g_{p} / d n\right)\right|_{n_{p}=n_{p_{-} t h}}}
$$

where $p=\mathrm{ES}$ or GS, $E_{p \text {,sat,gain }}$ is the saturation energy for the gain section, $E_{p, s a t, S A}$ is the saturation energy for the SA and $n_{p_{-} t h}$ is the corresponding carrier density at threshold.

For ML operation, the $S$ parameter should be larger than one, indicating that the gain section is harder to be saturated than the SA [16]; moreover, the increase of this parameter will benefit the ML stability as well as the obtained pulse quality [15]. Using (3) and referring to Fig. 2(b), we predict that GS ML could be established more easily and with an improved stability respect to ES ML, since $S_{G S}$ is always much larger than $S_{E S}$.

\section{B. Transient Responses to External Optical Excitations}

To complete the preliminary analysis, the transient responses of a gain section, acting as a semiconductor optical amplifier (SOA), and a reverse biased SA to an externally injected optical pulse are studied using the approaches described in [17]. Been $\Gamma$ the field confinement factor, in this work we will indicate with $\Gamma \Delta g_{m \leftarrow n}$ the time-dependent modal gain compression from $m=$ GS, ES caused by a pump pulse resonant with the $n=\mathrm{GS}$, ES transition. Similarly, the timedependent modal absorption bleaching will be $\Gamma \Delta \alpha_{m \leftarrow n}$. 

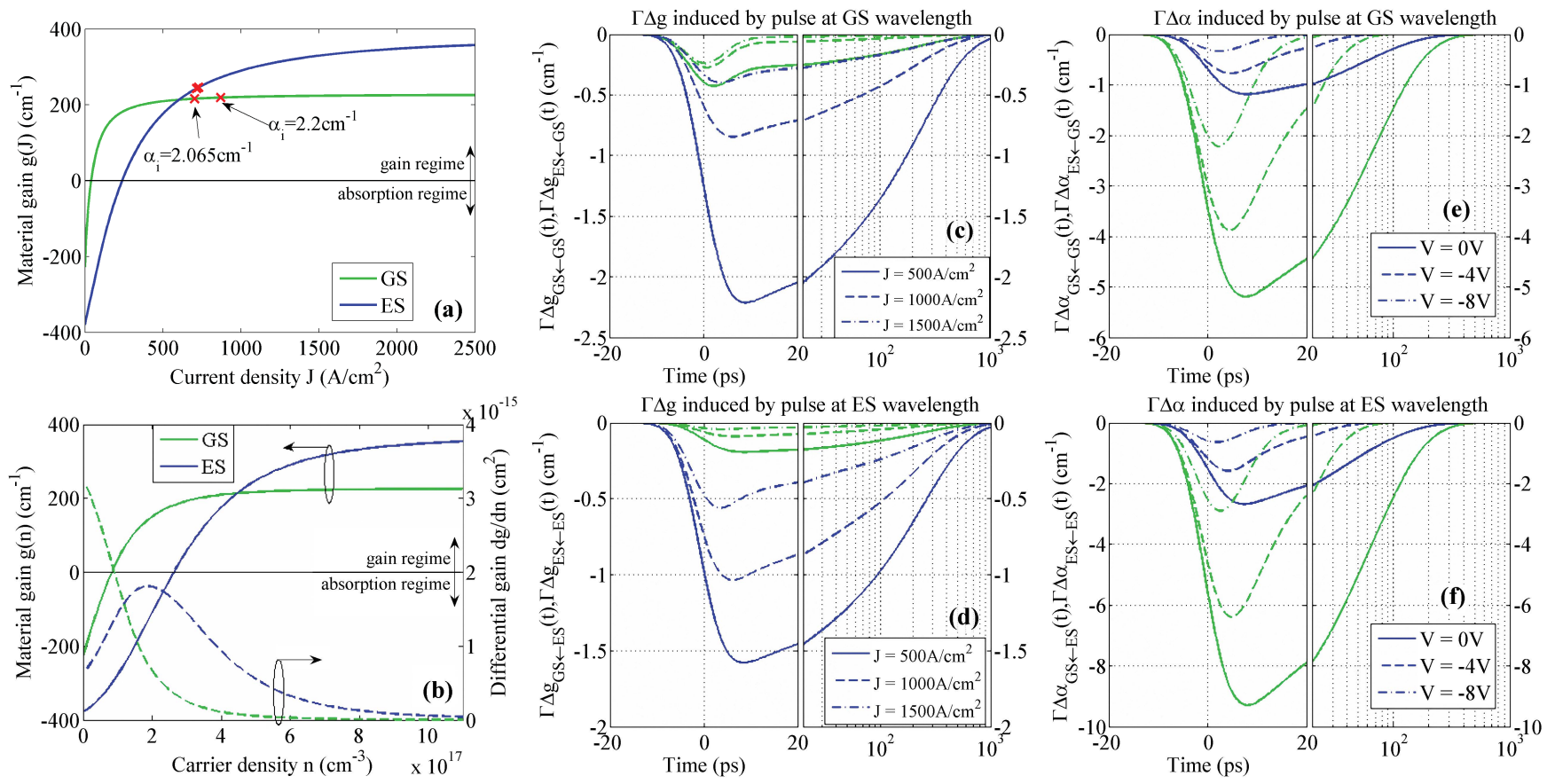

Fig. 2. (a) Steady state behavior of QD material gain from GS (green) and ES (blue) transitions as a function of the injection current density J. The red-cross markers represent the corresponding GS and ES threshold gain when intrinsic losses equal to $2.065 \mathrm{~cm}^{-1}$ and $2.2 \mathrm{~cm}^{-1}$, respectively, which will be discussed in Fig. 3. The horizontal black line, located at gain equals to $0 \mathrm{~cm}^{-1}$, distinguishes the gain and absorption regimes of the active medium. (b) Steady state behavior of material gain (solid line) and differential gain (dashed line) from GS (green) and ES (blue) transitions as a function of the averaged carrier density $\mathrm{n}$ in the DWELL layers. (c) Temporal evolution of GS (green) and ES (blue) modal gain compressions induced by a Gaussian pulse with 1 pJ energy, 10-ps duration, resonant with GS transition and traveling in a SOA biased with current density of $500 \mathrm{~A} / \mathrm{cm}^{2}$ (solid line), $1000 \mathrm{~A} / \mathrm{cm}^{2}$ (dashed line), and $1500 \mathrm{~A} / \mathrm{cm}^{2}$ (dotted-dashed line). Time interval $-20 \mathrm{ps}<\mathrm{t}<20 \mathrm{ps}$ is shown in the linear scale, whereas time interval $20 \mathrm{ps}<\mathrm{t}<1000 \mathrm{ps}$ is shown in the logarithmic scale. (d) Same quantities as in (c) induced by a Gaussian pulse with the same properties but resonant with ES transition. (e) Temporal evolution of the absorption bleaching induced by a Gaussian pulse resonant with GS transition and traveling in a SA with 0 V (solid line), -4 V (dashed line), and $-8 \mathrm{~V}$ (dotted line) reverse bias. (f) As in (e) but for a Gaussian pulse resonant with ES transition.

In Fig. 2(c) and Fig. 2(e), we report respectively the temporal evolutions of the modal gain compression $\left(\Gamma \Delta g_{G S \leftarrow G S}, \Gamma \Delta g_{E S \leftarrow G S}\right)$ and the modal absorption bleaching $\left(\Gamma \Delta \alpha_{G S \leftarrow G S}, \Gamma \Delta \alpha_{E S \leftarrow G S}\right)$, induced by a Gaussian pulse with $1 \mathrm{pJ}$ energy, $10 \mathrm{ps}$ duration (typical value for QD ML pulses) and resonant with the GS transition. On the contrary, the dynamics of the same quantities induced by a pulse with the same properties, but resonant with the ES transition $\left(\Gamma \Delta g_{G S \leftarrow E S}, \Gamma \Delta g_{E S \leftarrow E S}, \Gamma \Delta \alpha_{G S \leftarrow E S}\right.$ and $\left.\Gamma \Delta \alpha_{E S \leftarrow E S}\right)$ are shown in Fig. 2(d) and Fig. 2(f) respectively. The considered SOA/SA waveguide has a $6 \mu \mathrm{m}$ ridge width and a short total length to avoid the influence of field propagation effects.

Increasing $J$ from 500 to $1500 \mathrm{~A} / \mathrm{cm}^{2}$, a significant decrease in the entire gain recovery time (from ns scale to approximately tens ps) and in the maximum gain compression $\left(\Gamma \Delta g_{G S \leftarrow G S}\right.$ and $\left.\Gamma \Delta g_{E S \leftarrow E S}\right)$ for both states is observed (Fig. 2(c) and (d)) due to the enhanced carrier relaxation from higher electrical pumping levels. In addition, ES gain compression is found always to be much larger than that of the GS when excited by a pulse with the same energy, which means the GS amplification is harder to saturate than the ES amplification.

Similarly, increasing reverse bias voltage from 0 to $-8 \mathrm{~V}$, a significant reduction in the absorption recovery time (from hundreds to few ps) and in the maximum absorption bleaching $\left(\Gamma \Delta \alpha_{G S \leftarrow G S}\right.$ and $\left.\Gamma \Delta \alpha_{E S \leftarrow E S}\right)$ for both states is also observed (Fig. 2(e) and Fig. 2(f)), due to the enhanced thermionic and tunneling carrier escape mechanisms in the SA [18]. Additionally, if biased with the same voltage, the absorber always shows larger absorption depletion in GS transition than in ES transition, which means ES absorption is harder to saturate if compared with GS absorption.

The dynamic properties described above confirm again that GS ML can be achieved more easily, since it is easier to obtain simultaneous pulse shortening and amplification mechanisms from GS transition.

The photon-generated carriers at ES are quickly swept out during the pump pulse (10 ps), leading to smaller absorption bleaching in ES. There are two main aspects responsible for this fast carrier sweep-out. One is the smaller intraband energy difference between ES and the separate confinement heterostructure level, thus carriers at ES are swept out faster via the tunneling escape. Another one, which is more important, is the extremely fast carrier relaxation from ES to the GS (hundreds fs), existing as an additional carrier sweep-out path. The latter aspect is referred to as the photon-pumping process in [19]. This process and the related fast carrier escape (about $1 \mathrm{ps)} \mathrm{from} \mathrm{GS} \mathrm{to} \mathrm{ES} \mathrm{guarantee} \mathrm{the} \mathrm{recovery}$ of a quasi-equilibrium distribution in the QD layers and are responsible for the associated transient changes in GS/ES induced by a pulse resonant with ES/GS. These dynamics imply the strong coupling between GS and ES gain/absorption 
saturations and are key effects in determining the lasing regimes.

For a typical single section laser, the modal gain is clamped at its threshold value when lasing is achieved. For the twosection passively ML laser, however, the absorption bleaching in the SA induced by the non-zero optical power after lasing onset, allows the device to operate at a modal gain which is even smaller than its threshold value. For example, if we assume the threshold current for ES lasing $I_{t h-E S}$ is larger than that for GS $I_{t h_{-} G S}$, GS lasing is achieved first. When changing the bias condition, this lasing can be sustained, if the photoninduced absorption bleaching $\Gamma \Delta \alpha_{p}$ and gain compression $\Gamma \Delta g_{p}$ ( $p=\mathrm{GS}$ in this case) balance the following equation:

$$
\begin{aligned}
\frac{L_{\text {gain }}}{L}\left(\Gamma g_{p_{-} t h}+\Gamma \Delta g_{p}\right) & -\frac{L_{S A}}{L}\left(\Gamma \alpha_{p_{-} t h}+\Gamma \Delta \alpha_{p}\right) \\
& -\alpha_{i}-\frac{1}{L} \ln \left(\frac{1}{\sqrt{R_{1} R_{2}}}\right)=0
\end{aligned}
$$

where $\Gamma g_{p_{-} t h}$ is the modal gain at the threshold current for the first lasing state (in this case the GS), $\Gamma \alpha_{p_{-} t h}$ is the modal loss at the same current, $L_{\text {gain }}$ is the length of the gain section, $L_{S A}$ is the length of the SA, $L$ is the total device length which equals to $L_{\text {gain }}+L_{S A}, \alpha_{i}$ is the cavity intrinsic losses, and $R_{1}$ and $R_{2}$ are the reflectivities at the SA and the gain section side facets respectively. One should note that, unlike the transient response $\Gamma \Delta g_{m \leftarrow n}, \Gamma \Delta g_{p}$ in (4) represents the modal gain changes at $p=\mathrm{GS}$, ES transition induced by the optical power (from both GS and ES) in the cavity, and averaged over time and the length of the gain section. Similarly, $\Gamma \Delta \alpha_{p}$ is the modal absorption changes averaged over time and the length of SA.

Obviously, ES round trip gain (4) is negative at GS threshold current $I_{t h \_G S}$. However, at higher current, if the GS photon-induced gain/absorption changes in the ES satisfy the condition

$$
\left|\Gamma \Delta g_{p}\right|<\left(L_{S A} / L_{\text {gain }}\right)\left|\Gamma \Delta \alpha_{p}\right|,
$$

there will be a positive change in the original ES overall round trip gain and therefore the onset of lasing from ES is facilitated. On the contrary, if the condition

$$
\left|\Gamma \Delta g_{p}\right|>\left(L_{S A} / L_{\text {gain }}\right)\left|\Gamma \Delta \alpha_{p}\right|
$$

holds, there will be a negative change in the round trip gain and therefore ES lasing will tend to be inhibited. Situations are the same if inversely ES lasing happens first.

From the above discussions and from Fig. 2(c) and Fig. 2(e), we find that if lasing from GS is achieved first, GS optical field will lead to similar level of changes in ES gain, $\Gamma \Delta g_{E S \leftarrow G S}=$ $-0.4 \sim-2.2 \mathrm{~cm}^{-1}$ and absorption, $\Gamma \Delta \alpha_{E S \leftarrow G S}=-0.3 \sim$ $-1.2 \mathrm{~cm}^{-1}$. ES lasing is therefore inhibited at certain bias conditions (satisfying (6)), since $L_{S A}$ is generally much shorter than $L_{\text {gain }}$ for QD passively ML laser $\left(L_{S A} / L_{\text {gain }}=\right.$ $0.1 \sim 0.25$ ).

On the contrary, if lasing from ES is achieved first, thanks to the very efficient photon-pumping process, the generated ES carriers quickly relax towards the GS, contributing to the large absorption saturation at the GS wavelength. Thus, appreciable reduction of GS saturable losses $\Gamma \Delta \alpha_{G S \leftarrow E S}$
$=-3 \sim-9 \mathrm{~cm}^{-1}$ with respect to the tiny small reduction of GS gain $\Gamma \Delta g_{G S \leftarrow E S}=-0.04 \sim-0.2 \mathrm{~cm}^{-1}$ (Fig. 2(d) and Fig. 2(f)) are observed, and lasing from GS is promoted (satisfying (5)). Therefore, we can conclude that lasing from GS tends to be achieved much more easily with respect to the lasing from ES in the two-section QD lasers. Note that these effects are more evident at low current and low SA voltage.

In the following sections, we show how the investigated static and dynamic properties of the gain and the absorption influence the achieved lasing and ML regimes in the InAs/InGaAs QD based devices.

\section{Sole GS ML AND DuAl-State ML}

We consider in this section two ridge-waveguide edgeemitting QD ML lasers with different intrinsic losses $\alpha_{i}$ of $2.065 \mathrm{~cm}^{-1}$ and $2.2 \mathrm{~cm}^{-1}$ to change the laser operation point on the gain characteristic curves. Both devices have a fixed total length of $2 \mathrm{~mm}$, absorber length $L_{S A}$ of $300 \mu \mathrm{m}$, ridge width of $6 \mu \mathrm{m}$, high reflection coating at the SA side facet with $R_{1 p}=99 \%$ ( $p=\mathrm{ES}$, GS) and low reflection coating at the output facet with $R_{2 p}=10 \%$. The parameters of the active medium are those considered in Section III.

\section{A. Lasing Regime Discussion}

For these two devices, in Fig. 3(a) and 3(b) respectively, maps of the achieved operating regimes are reported in the injection current and reverse bias voltage plane, summarizing the corresponding lasing state at each bias point. In addition, in Fig. 2(a), the associated threshold material gains for the lasing from GS $\left(g_{t h_{-} G S}\right)$ and ES $\left(g_{t h_{-} E S}\right)$ transitions of the same devices are shown as red-cross markers on the corresponding gain curves. The threshold material gain can be computed following (4) by setting $\Gamma \Delta g_{p}$ and $\Gamma \Delta \alpha_{p}$ to zero.

With increasing current, $g_{G S}$ shows earlier saturation than $g_{E S}$, therefore a slight increase in the intrinsic losses leads to a large increase in the GS threshold current $I_{t h_{-} G S}$, whereas for the ES, threshold current $I_{t h \_E S}$ doesn't show severe increase due to the larger $d g / d J$. More precisely, the computed results show that $I_{t h} G S<I_{t h-E S}$ when $\alpha_{i}=2.065 \mathrm{~cm}^{-1}$ and $I_{t h_{-} G S}>I_{t h_{-} E S}$ when $\alpha_{i}=2.2 \mathrm{~cm}^{-1}$ (Fig. 2(a)).

The relative relationships between $I_{t h_{-} G S}$ and $I_{t h-E S}$ are the key reasons governing the achieved lasing regimes in Fig. 3(a) and $3(\mathrm{~b})$. Furthermore, in order to get more insight on the physical origin of the different lasing regimes, the time-spaceaveraged occupation probabilities $\rho$ in SA and gain sections of GS and ES at several fixed reverse voltages are depicted in Fig. 3(c)-(f) as a function of the injection current.

For the first device (Fig. 3(a)), $I_{t h} G S$ is slightly smaller than $I_{t h} E S$, therefore GS lasing is achieved first; the selfconsistent solution of the DDE equations and rate-equation system for GS is obtained in a large range of bias conditions above the first lasing threshold $I_{t h} G S$. The balance between the occupation probability changes in the SA $\Delta \rho_{S A}$ and the gain section $\Delta \rho_{\text {gain }}$ is observed when increasing current above the threshold, always satisfying (4). This can be verified by substituting in (4) the following equations: $\Gamma \Delta g=2 \Gamma g_{0}$ * $\Delta \rho_{\text {gain }}$ and $\Gamma \Delta \alpha=-2 \Gamma g_{0} * \Delta \rho_{S A}$, being $g_{0}$ the material 

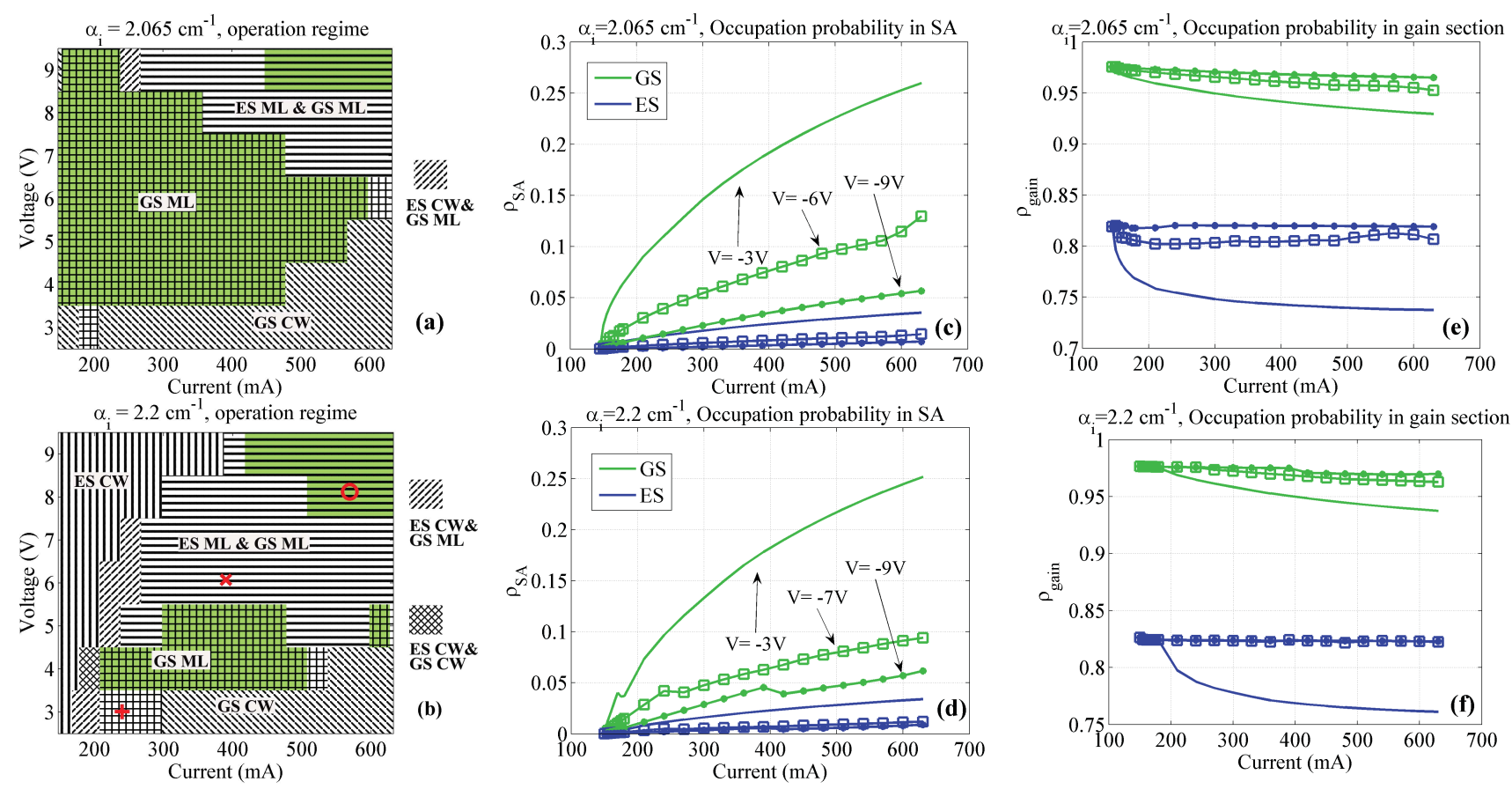

Fig. 3. Maps of the operation regime as a function of the injection current for the gain section and the reverse bias voltage applied to the SA for QD two-section ML laser with intrinsic losses of (a) $2.065 \mathrm{~cm}^{-1}$ and (b) $2.2 \mathrm{~cm}^{-1}$. There are two layers in each figure; the background layer distinguishes the bias condition region that achieves stable ML regime with green color; the top layer indicates various operation regimes by filling the corresponding bias condition region with different line styles: GS continuous wave (GS CW, forward diagonal line), ES continuous wave (ES CW, vertical line), simultaneous GS and ES continuous wave (ES CW \& GS CW, diagonal cross), GS mode locking and ES continuous wave (ES CW \& GS ML, backward diagonal line), sole GS mode locking (GS ML, large grid), simultaneous GS mode locking, and ES mode locking (ES ML \& GS ML, horizontal line). Cross, circle, and plus markers in (b) identify the operation points which will be discussed later. Time-space-averaged occupation probability in the (c) SA and (e) gain section of the GS (green) and ES (blue) for a QD ML laser with intrinsic losses of $2.065 \mathrm{~cm}^{-1}$ when SA reverse bias voltage is $-3 \mathrm{~V}$ (solid line), $-6 \mathrm{~V}$ (square marked), and $-9 \mathrm{~V}$ (star marked). Time-space-averaged occupation probability in the (d) SA and (f) gain section of the GS (green) and ES (blue) for a QD ML laser with intrinsic losses of $2.2 \mathrm{~cm}^{-1}$ when SA reverse bias voltage is $-3 \mathrm{~V}$ (solid line), $-7 \mathrm{~V}$ (square marked), and $-9 \mathrm{~V}$ (star marked).

gain coefficient. We can see from Fig. 3(c) and Fig. 3(e) that, $\Delta \rho_{S A}$ and $\Delta \rho_{\text {gain }}$ of GS always obey the rule that $\left|\Delta \rho_{\text {gain }}\right|=$ $\left|\Delta \rho_{S A}\right| * L_{S A} /\left(L-L_{S A}\right)\left(L_{S A}=0.3 \mathrm{~mm}\right.$ is the $\mathrm{SA}$ length, and $L-L_{S A}=1.7 \mathrm{~mm}$ is the gain section length), preserving the lasing from GS. In addition, consistently with the information obtained in Section III, due to the optical power from GS, lasing from ES is prevented, especially at low reverse bias voltage $(-3 \mathrm{~V}$ and $-6 \mathrm{~V})$. As an evidence, there is a large reduction in $\rho_{\text {gain }}$ but only a slight increase in $\rho_{S A}$ for the ES, which satisfies the condition (6). By increasing the reverse voltage, due to the enhanced absorption recovery rate (Fig. 2(e)) and the reduced GS optical power, maximum GS absorption bleaching (Fig. 3(c)) decreases severely. To guarantee unitary round trip gain, the corresponding GS gain compression also decreases (Fig. 3(e)), therefore the associated $\Delta \rho_{\text {gain }}$ of ES becomes negligible. Under this condition, if we increase also the injection current, GS pulse energy will increase, leading to larger ES absorption bleaching. At the same time, negligible ES gain compression is still guaranteed due to enhanced carrier pumping process at high current level. So finally, onset of ES lasing can be achieved when ES absorption bleaching is high enough to compensate the original negative total cavity gain of ES. Furthermore, increasing the reverse voltage, since the ES gain compression decreases, the needed reduction level of the ES absorption also decreases; consequently the onset current of ES lasing is shifted toward lower current.
The numerically obtained operation regimes in the first device is qualitatively consistent with the experimental observations in [8], where coexisting ES and GS lasing are achieved at high injection current and high reverse bias voltage. In addition, the onset current of ES lasing is shown to decrease, though not monotonically, with increasing the reverse voltage.

On the contrary, for the second laser with $\alpha_{i}=2.2 \mathrm{~cm}^{-1}$ (Fig. 3(b)), $I_{t h_{-} E S}$ is now smaller than $I_{t h_{-} G S}$ (see Fig. 2(a)), ES lasing is therefore always achieved first. In this case, GS lasing can not be self-starting. However, as we mentioned in Section III, optical power from ES will leads to severe reduction in the GS absorption (Fig. 3(d)) due to the fast relaxation of the photon-generated ES carriers towards the GS in the SA. Furthermore, a relatively smaller compression of GS gain (Fig. 3(f)) is obtained. These two changes easily satisfy (5) and therefore reduce GS round trip cavity losses; this effect becomes stronger at larger current since ES power becomes larger. Therefore, when the current is sufficiently high, GS lasing turns on. Additionally, increasing the reverse voltage, photon-generated carriers at ES are swept away more efficiently via the enhanced thermionic and tunneling escape processes. As a result, the amount of carriers that relax from ES to GS reduces significantly, leading to a reduction in the effectiveness of the ES induced positive compensation of GS total cavity losses. Consistently with this, in Fig. 3(b), onset 
current of GS lasing is postponed to higher value when SA voltage increases.

Once the lasing from GS is achieved, the operation conditions will become similar to those of the first device. At lower voltage, see example in Fig. 4, ES emission starts first, leading to large GS absorption bleaching in the SA and favoring the lasing from GS. Once GS lasing starts, thanks to the already bleached SA absorption, GS lasing can be self-sustained even without the ES emission. In addition, ES lasing is prevented for the reasons described in Section III and previously for the first device. This can be validated by the large gain compression and relative small absorption bleaching of ES when $V=$ $-3 \mathrm{~V}$ (Fig. 3(d) and Fig. 3(f)), which is exactly the same situation that happens in the first device. Based on the same reason, at larger reverse voltage, dual-state lasing is obtained.

The simulated operation regimes for this device is instead shown to be in qualitatively agreement with the experimental results in [7], where reverse-emission-state-transition, i.e., ES lasing is achieved first and then followed by GS lasing at higher current, is observed. As we discussed here, the onset of GS lasing is shifted toward larger current when increasing the reverse voltage.

\section{B. ML Performances Discussion}

We take the device with $\alpha_{i}=2.2 \mathrm{~cm}^{-1}$ as an example for further investigations on the ML characteristics. The maps of peak power and pulse width of ML regimes from GS and ES are reported in Fig. 5(a)-(d) in a plane of the forward injection current and the reverse bias voltage.

From Fig. 3(b), we can find that a variety of ML regimes are achieved in this device. It is noteworthy to point out that, although sole ES lasing is obtained in a large range of bias conditions, sole ES ML is never observed. The restrictions to achieve sole ES ML will be discussed in detail in Section V. Consistently with the commonly observed experimental performances for the typical GS ML QD lasers [1], the maximum GS peak power and the minimum GS pulse width are observed at low current and high voltage when sole GS ML is achieved (Fig. 5(a) and Fig. 5(c)). Just beyond the sole GS ML region, at higher current, the leading edge instability (LEI) induced by spontaneous emission noise is encountered, which finally results in continuous wave operation of the GS.

On the contrary, within dual-state ML region, in most of the bias conditions, an unstable dual-state ML with regular amplitude modulation is observed (Fig. 3(b)), with similar pulse width and peak power as sole GS ML (Fig. 5(a) and Fig. 5(c)). Nevertheless, it is noticeable that at high current and high voltage, a stable dual-state ML regime is achieved, accompanied with remarkable enhanced pulse peak power and pulse width for both ES and GS. This stable ML regime can be obtained only in limited bias conditions, since at higher current (beyond the bias condition in Fig. 3) the LEI will start to appear first in the GS ML. Two operating conditions, one representing the unstable dual-state ML (the cross marker, $\mathrm{V}=-6 \mathrm{~V}, \mathrm{I}=390 \mathrm{~mA}$ ) and another representing the stable dual-state ML (the circle marker, $\mathrm{V}=-8 \mathrm{~V}, \mathrm{I}=570 \mathrm{~mA}$ ), are studied in detail later.

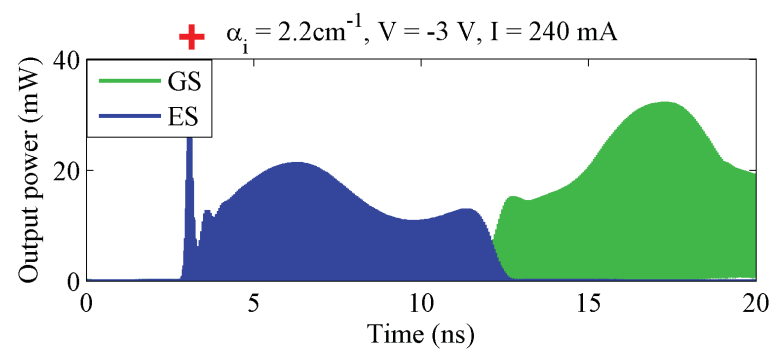

Fig. 4. Time trace of the output peak power of GS (green) and ES (blue) at the initial 20-ns time interval of the simulation for the device shown in Fig. 3(b) when injection current is $240 \mathrm{~mA}$ and reverse voltage is $-3 \mathrm{~V}$ [the plus marker in Fig. 3(b)].

In Fig. 5(e) and Fig. 5(f), the radio frequency (RF) spectra of these two conditions are reported in a narrow frequency window around the fundamental repetition frequency. For the first operating condition, two distinct fundamental lines appear in the RF spectrum (Fig. 5(e)), corresponding respectively to the pulse train of GS with a repetition frequency $f_{G S}=$ $22.374 \mathrm{GHz}$ and the pulse train of ES with a repetition frequency $f_{E S}=21.915 \mathrm{GHz}$. This difference in the repetition frequencies confirms the different group velocities of GS and ES pulses. As shown in Fig. 6, this difference not only leads to desynchronization between GS and ES pulse trains, but also results in an amplitude modulation characterized by a periodicity of $1 / \Delta f\left(\Delta f=f_{G S}-f_{E S}=459 \mathrm{MHz}\right)$. In frequency domain, this is equivalent to multiple side-bands around the fundamental GS and ES lines with lower power and equal separation $\Delta f$ between each other. This RF spectrum shows qualitative agreement with the experimental observations in [7], where both the fundamental lines and the side-bands are present when dual-state ML is achieved. On the contrary in [8], although dual-state ML is also observed, the achieved ES pulse power at the shown bias condition is very small with respect to the GS pulse power, extremely weakening the influence of the ES pulse on the GS pulse. As a consequence, the side-bands would have an even smaller power that is almost merged with the background noise. We believe this fact explains partially the absence of side lobes in the reported RF spectrum in [8]. We also observed that, in the simulations, the strength of this amplitude modulation and the side-band/main-peak power ratio change with the bias condition.

A completely different dynamic regime is instead found at the second operating point, where a single well defined line at $f_{G S}$ is observed in the RF spectrum (Fig. 5(f)), indicating that the pulses from both states are propagating with the same velocity $v_{g_{-} G S}\left(v_{g_{-} G S}>v_{g_{-} E S}\right)$ in the cavity. We find that this locked traveling of ES and GS pulses arises from the large forward shift of the ES pulse in time domain induced by the large positive net gain window preceding the pulse.

To clarify the physical origins of the amplitude modulation operation in the first condition and the locked pulse traveling in the second condition, the associated gain and absorption dynamics determining the generation of the mode locked pulses are depicted in Fig. 6 and Fig. 7. In these figures, the total losses $A(\tau)$ and the total amplification $G(\tau)$ experienced 

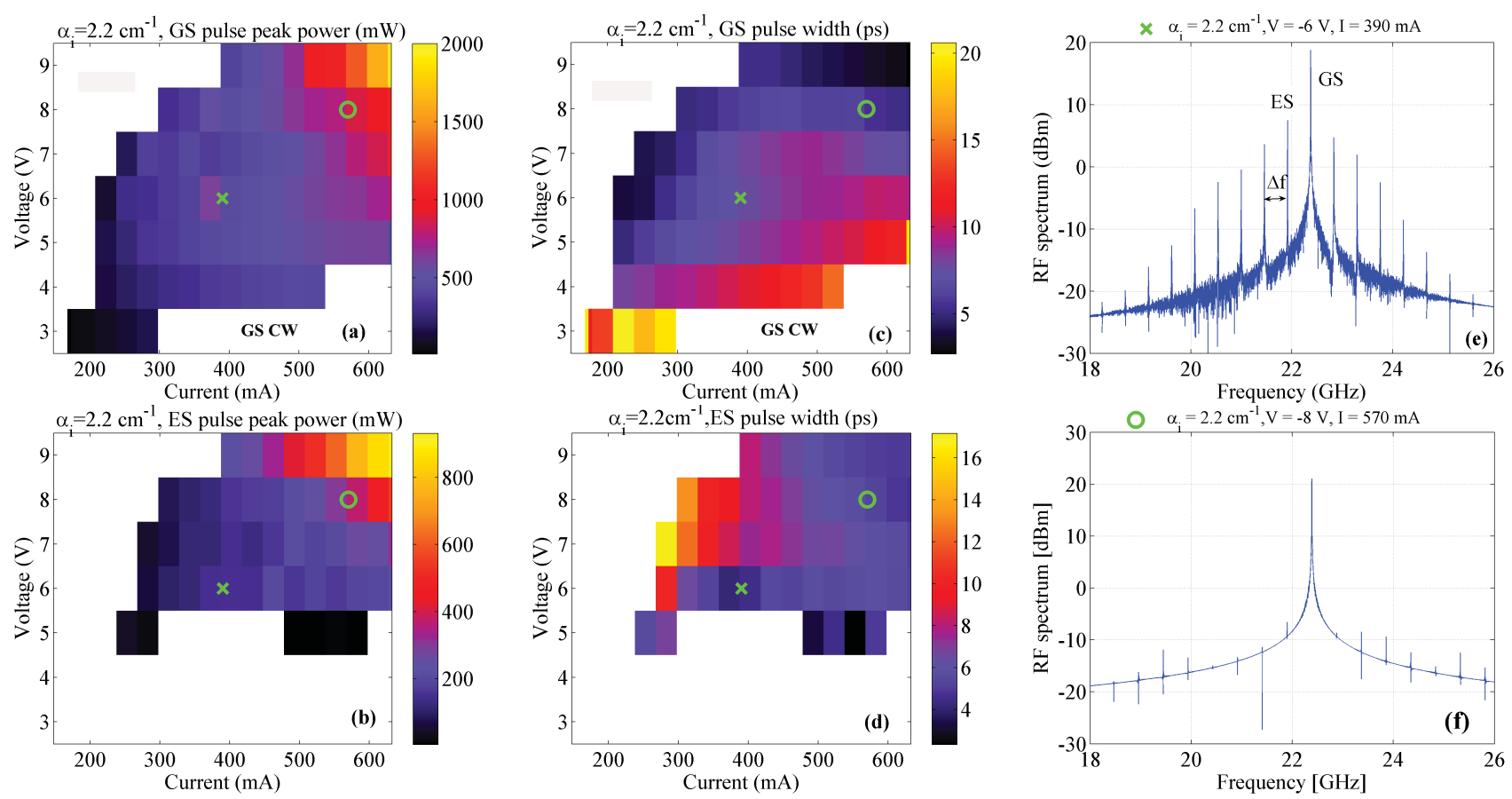

Fig. 5. Maps of (a) GS pulse peak power, (b) ES pulse peak power, (c) GS pulse width, and (d) ES pulse width as a function of the injection current and reverse voltage for the device with intrinsic losses is $2.2 \mathrm{~cm}^{-1}$ as shown in Fig. 3(b). For same device, the RF spectra of the pulse trains when the laser is (e) biased with $V=-6 \mathrm{~V}, I=390 \mathrm{~mA}$ and (f) biased with $V=-8 \mathrm{~V}, I=570 \mathrm{~mA}$, corresponding to the cross and circle markers shown in Fig. 5 (a) and (b), respectively.

by the pulse during one complete round trip within the laser cavity are shown in detail.

Consistently with the presence of side-bands in the RF spectrum envelope, amplitude modulations are observed in the time domain pulse traces of GS and ES (Fig. 6(a)) with a characteristic periodicity $1 / \Delta f$. Pulse evolutions and the associated $G(\tau)$ and $A(\tau)$ dynamics in two distinct short time intervals are zoomed in Fig. 6(c)-(d) and Fig. 6(e)-(g) respectively, corresponding to the highlighted region 1 and region 2 in Fig. 6(a).

In the first time interval, ES pulse follows the GS pulse with a large relative delay. As discussed previously, GS lasing is always self-preserved and immediately leads to a significant reduction of the ES gain $G(\tau)$ (Fig. 6(d)). However, this GS pulse induced ES gain reduction now has enough time to recover back to a sufficient high value that overcomes the overall ES losses $A(\tau)$ before the arrival of the subsequent ES pulse. Therefore, ES pulse feels a positive net gain within one round trip and tends to be amplified. In addition, ES gain reduction induced by the ES pulse is compensated by the carriers escaped from GS state, leading to an early started compression of the GS gain (Fig. 6(c)), which is encountered by the closely followed subsequent GS pulse, resulting in a gradual decrease in the GS pulse energy.

Due to the different group velocity, the relative delay between two adjacent GS and ES pulses increases with time in region 1 and finally ES pulse is overlapped with the followed GS pulse in the region 2 (Fig. 6(e)). In this case, the GS pulse induces a large ES gain compression experienced by the synchronous ES pulse, as a result, ES pulse has a negative round trip gain and consequently its energy decreases gradually (Fig. 6(g)). On the contrary, the ES carriers produced by the absorption of ES pulse in the SA, quickly pump the lower energy GS, reducing significantly the GS absorption seen by the synchronous GS pulse. Due to this effect, positive round trip gain is achieved in GS, enhancing gradually GS pulse energy (Fig. 6(f)).

In [7], dual-stable ML is achieved at rather low reverse voltage (even at $0 \mathrm{~V}$ ) and thus the interplays between GS and ES pulses are dominated by the absorption dynamics in the SA. Here, on the contrary the photon pumping process is much less efficient since device operate at very high reverse voltage and instead the coupled GS and ES gain dynamics in the gain section are instead dominating. This can be clarified by the fact that the increase or decrease of ES pulse energy is achieved at different relative time-location of ES and GS pulses with respect to that reported in [7].

For the second operating condition, well-formed pulse traces from GS and ES with negligible peak power jitter can be seen in Fig. 7(a). The behavior of GS pulse can be easily understood by analyzing the evolutions of $G(\tau)$ and $A(\tau)$ in Fig. 7(b). The overall round trip gain $G(\tau)$ equals exactly the overall losses $A(\tau)$ when there is the pulse, guaranteeing an identical reproduced pulse after one round trip. Being $G(\tau)$ smaller than $A(\tau)$ behind the pulse, the noise perturbations following the pulse are eliminated, whereas the tiny positive net gain window before the pulse is not strong enough to lead to the LEI; therefore stable GS ML is sustained.

A very different ES net gain window with respect to that shown in Fig. 6 is observed in the second operating condition. In Fig. 6(d) and Fig. 6(g), ES pulse's leading edge and trailing edge experience simultaneous overall attenuation or amplification. Therefore, there is no relative shift of the pulse in time domain when circulating in the cavity and 

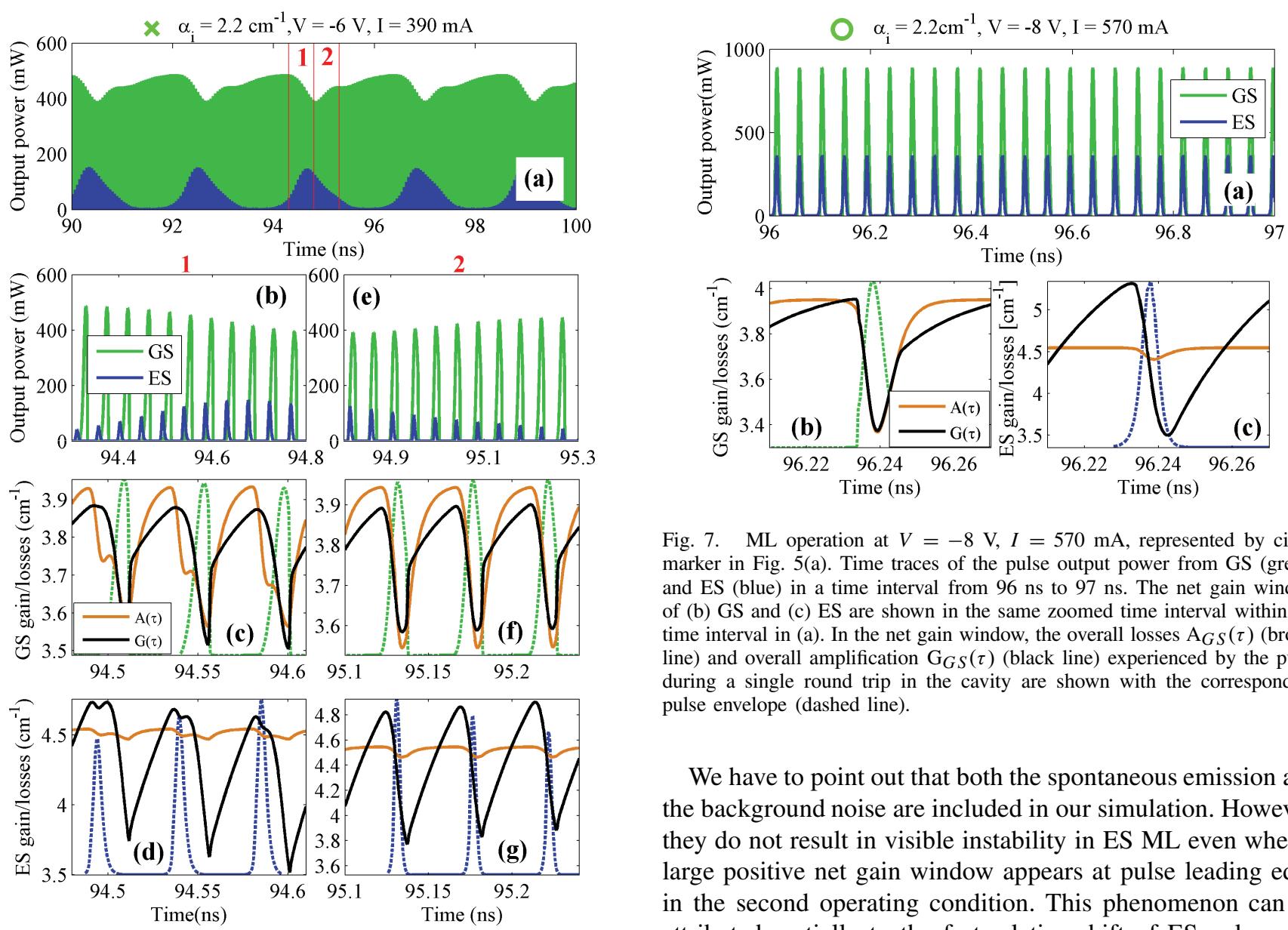

Fig. 6. ML operation at $\mathrm{V}=-6 \mathrm{~V}, \mathrm{I}=390 \mathrm{~mA}$, represented by cross marker in Fig. 5. (a) Time traces of the pulse output power from GS (green) and ES (blue) in a time interval from $90 \mathrm{~ns}$ to $100 \mathrm{~ns}$. Output power from (b) GS and ES, and the net gain window of (c) GS and (d) ES are shown in the zoomed time interval corresponding to the highlighted region 1 in (a). Output power from GS and (e) ES, and the net gain window of (f) GS and (g) ES are shown in the zoomed time interval corresponding to the highlighted region 2 in (a). In the net gain window, the overall losses $\mathrm{A}_{G S}(\tau)$ (brown line) and overall amplification $\mathrm{G}_{G S}(\tau)$ (black line) experienced by the pulse during a single round trip in the cavity are shown with the corresponding pulse envelope (dashed line).

ES pulse travels with its own group velocity $v_{g_{-} E S}$. In the second case, however, a positive/negative net gain window appears before/after ES pulse peak (Fig. 7(c)), contributing to an amplification of the leading edge optical field and an attenuation of the trailing edge optical field. Influenced by the combination of these two effects, ES pulse is forward shifted in the time domain, equivalent to say that it is propagating with a larger group velocity. Actually, this larger group velocity is equal exactly to $v_{g_{-} G S}$ because, if a slight shift from $v_{g_{-} G S}$ happens, the GS pulse induced gain and absorption changes will force the ES pulse to go back. Therefore, ES pulse seems to be clamped with the pulse from GS in this case.

This equal-group-velocity dual-state ML has not been reported in literatures so far. To further validate the results, a reduced set of simulations in similar bias conditions have been done using TDTW model and the reported DDE results were fully confirmed.

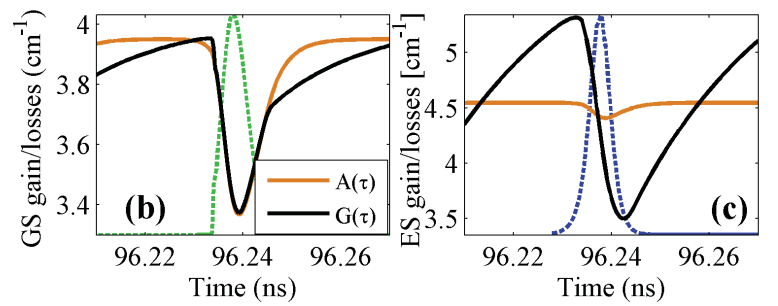

Fig. 7. ML operation at $V=-8 \mathrm{~V}, I=570 \mathrm{~mA}$, represented by circle marker in Fig. 5(a). Time traces of the pulse output power from GS (green) and ES (blue) in a time interval from $96 \mathrm{~ns}$ to $97 \mathrm{~ns}$. The net gain window of (b) GS and (c) ES are shown in the same zoomed time interval within the time interval in (a). In the net gain window, the overall losses $\mathrm{A}_{G S}(\tau)$ (brown line) and overall amplification $\mathrm{G}_{G S}(\tau)$ (black line) experienced by the pulse during a single round trip in the cavity are shown with the corresponding pulse envelope (dashed line).

We have to point out that both the spontaneous emission and the background noise are included in our simulation. However, they do not result in visible instability in ES ML even when a large positive net gain window appears at pulse leading edge in the second operating condition. This phenomenon can be attributed partially to the fast relative shift of ES pulse with respect to the background noise, which limits the amplification of the noise before being merged with the pulse [17].

\section{SOLE ES ML}

It can be seen from Fig. 3(a) and 3(b) that sole GS ML is established almost immediately after the onset of GS lasing. Then it is important to identify the reason why the sole ES ML cannot instead be achieved in the device with $\alpha_{i}=2.2 \mathrm{~cm}^{-1}$ at any of the investigated bias conditions and only continuous wave ES lasing is observed. Two main reasons are responsible for this result.

In Section III, the $S$ parameter has been introduced. For the device with $\alpha_{i}=2.2 \mathrm{~cm}^{-1}$, GS lasing happens first, so $d g_{G S} / d n$ and $d g_{E S} / d n$ are calculated at the threshold carrier density of the GS $n_{G S_{-} t h}$; and the computed $S$ parameter using (3) is 95 and 0.84 for GS and ES respectively. Therefore, it is clear that sole ES ML cannot be obtained in this device, since ES $S$ is less than one in this case, which means that the gain section is even easier to be saturated than the SA for ES.

Besides the requirement in the $S$ parameter, enough pulse energy to saturate the SA is also essential to form a pulse. For pulses longer than the time scale governing the recovery of a quasi-equilibrium distribution in the QD layers, the absorber saturation energy $E_{p, s a t, S A}(p=\mathrm{GS}$ or ES) can be estimated by the following equation [20]:

$$
E_{p, s a t, S A}=\frac{\hbar \omega_{p} W N_{l} h_{Q D}}{\left.\Gamma\left(d g_{p} / d n\right)\right|_{n_{p}=0}}
$$


where $\hbar \omega$ is the interband transition energy, $W$ is the ridge width, $N_{l}$ is the QD layer number, $h_{Q D}$ the height of a single QD layer.

According to Fig. 2(b) and (7), $E_{G S, s a t, S A}$ is found to be only $1.4 \mathrm{pJ}$ while $E_{E S, s a t, S A}$ is found to be approximately $7 \mathrm{pJ}$. Consistently with above values, GS ML happens almost immediately above its lasing threshold, while sole ES ML is not achieved since the onset of the ES-induced GS lasing requires a ES optical energy which is even smaller than $E_{E S, s a t, S A}$. Therefore, when current increases, the maximum accumulated ES optical energy before the onset of GS lasing is still not high enough to sufficiently saturate the ES absorption.

\section{CONCLUSION}

The competition between GS and ES ML regimes in two passively mode locked lasers has been simulated with a modified MS-DDE approach. The reported operations have been fully explained from the results of the investigations of the gain and absorption steady-state and dynamic properties for InAs/InGaAs QD medium.

Regarding the ML quality, we demonstrated not only the experimentally observed unstable dual-state ML, but also a stable dual-state ML regime at high injection current and reverse voltage, with significant improved peak power and pulse width. Simulations using TDTW model were used to validate the DDE model results.

\section{ACKNOWLEDGMENT}

The authors gratefully acknowledge the interesting discussions with M. A. Cataluna.

\section{REFERENCES}

[1] M. G. Thompson, A. R. Rae, M. Xia, R. V. Penty, and I. H. White, "InGaAs quantum-dot mode-locked laser diodes," IEEE J. Sel. Topics Quantum Electron., vol. 15, no. 3, pp. 661-672, May-Jun. 2009.

[2] E. A. Viktorov, M. A. Cataluna, L. O'Faolain, T. F. Krauss, W. Sibbett, E. U. Rafailov, and P. Mandel, "Dynamics of a two-state quantum dot laser with saturable absorber," Appl. Phys. Lett., vol. 90, no. 12, pp. 121113-121114, Jun. 2007.

[3] D. I. Nikitichev, Y. Ding, M. Ruiz, M. Calligaro, N. Michel, M Krakowski, I. Krestnikov, D. Livshits, M. A. Cataluna, and E. U. Rafailov, "High-power passively mode-locked tapered InAs/GaAs quantum-dot lasers," Appl. Phys. B, Lasers Opt., vol. 103, no. 3, pp. 609-613, 2011.

[4] J. Liu, Z. Lu, S. Raymond, P. J. Poole, P. J. Barrios, and D. Poitras, "Dual-wavelength $92.5 \mathrm{GHz}$ self-mode-locked InP-based quantum dot laser," Opt. Lett., vol. 33, no. 15, pp. 1702-1704, 2008.

[5] L. Olejniczak, K. Panajotov, S. Wieczorek, H. Thienpont, and M. Sciamanna, "Intrinsic gain switching in optically injected quantum dot laser lasing simultaneously from the ground and excited state," J. Opt. Soc. Amer. B, vol. 27, no. 11, pp. 2416-2423, 2010.

[6] S. Breuer, M. Rossetti, W. Elsässer, and L. Drzewietzki, "Reverseemission-state-transition mode locking of a two-section InAs/InGaAs quantum dot laser," Appl. Phys. Lett., vol. 97, no. 7, pp. 071118-1071118-3, 2010.

[7] M. Rossetti, L. Drzewietzki, P. Bardella, I. Montrosset, and W. Elsasser, "Joint experimental and theoretical investigations of two-state mode locking in a strongly chirped reverse-biased monolithic quantum dot laser," IEEE J. Quantum Electron., vol. 47, no. 10, pp. 1320-1329, Oct. 2011.

[8] M. A. Cataluna, D. I. Nikitichev, S. Mikroulis, H. Simos, C. Simos, C. Mesaritakis, D. Syvridis, I. Krestnikov, D. Livshits, and E. U. Rafailov, "Dual-wavelength mode-locked quantum-dot laser, via ground and excited state transitions: Experimental and theoretical investigation," Opt. Exp., vol. 18, no. 12, pp. 12832-12838, 2010.
[9] M. Rossetti, P. Bardella, and I. Montrosset, "Modeling passive modelocking in quantum dot lasers: A comparison between a finite-difference traveling-wave model and a delayed differential equation approach," IEEE J. Quantum Electron., vol. 47, no. 5, pp. 569-576, May 2011.

[10] M. Rossetti, P. Bardella, and I. Montrosset, "Time-domain travellingwave model for quantum dot passively mode-locked lasers," IEEE $J$ Quantum Electron., vol. 47, no. 2, pp. 139-150, Feb. 2011.

[11] A. G. Vladimirov and D. Turaev, "Model for passive mode locking in semiconductor lasers," Phys. Rev. A, vol. 72, no. 3, pp. 033808-033821, 2005.

[12] J. G. Bresco, S. Dommers, V. V. Temnov, U. Woggon, J. Martinez-Pastor, M. Laemmlin, and D. Bimberg, "InGaAs quantum dots coupled to a reservoir of nonequilibrium free carriers," IEEE J. Quantum Electron., vol. 45, no. 9, pp. 1121-1128, Sep. 2009.

[13] P. Borri, W. Langbein, J. M. Hvam, F. Heinrichsdorff, M. H. Mao, and D. Bimberg, "Spectral hole burning and carrier heating dynamics in InGaAs quantum dots amplifiers," IEEE J. Sel. Topics Quantum Electron., vol. 6, no. 3, pp. 544-551, May-Jun. 2000.

[14] H. Haus, "Theory of mode locking with a slow saturable absorber," IEEE J. Quantum Electron., vol. 11, no. 9, pp. 736-746, Sep. 1975.

[15] Y. C. Xin, Y. Li, V. Kovanis, A. L. Gray, L. Zhang, and L. F. Lester, "Reconfigurable quantum dot monolithic multi-section passive modelocked lasers," Opt. Exp., vol. 15, no. 12, pp. 7623-7633, 2007.

[16] R. G. M. P. Koumans and R. V. Roijen, "Theory for passive modelocking in semiconductor laser structures including the effects of selfphase modulation, dispersion, and pulse collisions," IEEE J. Quantum Electron., vol. 32, no. 3, pp. 478-492, Mar. 1996.

[17] M. Rossetti, T. Xu, P. Bardella, and I. Montrosset, "Impact of gain saturation on passive mode locking regimes in quantum dot lasers with straight and tapered waveguides," IEEE J. Quantum Electron., vol. 47, no. 11, pp. 1404-1413, Nov. 2011.

[18] A. Gomez-Iglesias, S. J. White, W. Sibbett, A. Miller, and E. U. Rafailov, "Ultrafast electroabsorption dynamics in an InAs quantum dot saturable absorber at $1.3 \mu \mathrm{m}, "$ Appl. Phys. Lett., vol. 89, no. 17, pp. 171111171113,2006

[19] A. Markus, M. Rossetti, V. Calligari, D. Chek-Al-Kar, J. X. Chen, A. Fiore, and R. Scollo, "Two-state switching and dynamics in quantum dot two-section lasers," J. Appl. Phys., vol. 100, no. 11, pp. 113104-1$113104-5,2006$

[20] A. V. Uskov, T. W. Berg, and J. Mørk, "Theory of pulse-train amplification without patterning effects in quantum-dot semiconductor optical amplifiers," IEEE J. Quantum Electron., vol. 40, no. 3, pp. 306-320, Mar. 2004.

Tianhong Xu received the B.Sc. degree in electronic science and technology from the Huazhong University of Science and Technology, Wuhan, China, in 2007, and the Laurea Magistrale degree in electronic and communication engineering from the Politecnico di Torino, Turin, Italy, in 2009, where she is currently pursuing the Ph.D. degree.

Her current research interests include modeling and simulation of quantumdot-based optoelectronic devices.

Mattia Rossetti received the Laurea Magistrale degree in physical engineering and the Ph.D. degree in electronic and communication engineering from the Politecnico di Torino, Turin, Italy, in 2007 and 2011, respectively.

His current research interests include modeling and simulation of electronic and optoelectronic devices.

Paolo Bardella received the Laurea degree in electronic engineering and the $\mathrm{Ph} . \mathrm{D}$. degree in electronic and communication engineering from the Politecnico di Torino, Turin, Italy, in 2001 and 2006, respectively.

$\mathrm{He}$ is currently a Post-Doctoral Fellow with the Dipartimento di Elettronica e Telecomunicazioni, Politecnico di Torino. His current research interests include modeling and design of multisection quantum-dot semiconductor lasers and superluminescent diodes.

Ivo Montrosset (M'92) has been a Professor of optoelectronics with the Politecnico di Torino, Turin, Italy, since 1990. His current research interests include guided wave-optics, solid-state and semiconductor lasers, and other related topics.

$\mathrm{He}$ is a member of the IEEE Photonics Society. 